\title{
Demonstration of a Fundamental Quantum Logic Gate
}

\author{
C. Monroe, D. M. Meekhof, B. E. King, W. M. Itano, and D. J. Wineland \\ National Institute of Standards and Technology, Boulder, Colorado 80303
}

(Received 14 July 1995)

\begin{abstract}
We demonstrate the operation of a two-bit "controlled-NOT" quantum logic gate, which, in conjunction with simple single-bit operations, forms a universal quantum logic gate for quantum computation. The two quantum bits are stored in the internal and external degrees of freedom of a single trapped atom, which is first laser cooled to the zero-point energy. Decoherence effects are identified for the operation, and the possibility of extending the system to more qubits appears promising.
\end{abstract}

PACS numbers: $89.80 .+\mathrm{h}, 03.65 .-\mathrm{w}, 32.80 . \mathrm{Pj}$

We report the first demonstration of a fundamental quantum logic gate that operates on prepared quantum states. Following the scheme proposed by Cirac and Zoller [1], we demonstrate a controlled-NOT gate on a pair of quantum bits (qubits). The two qubits comprise two internal (hyperfine) states and two external (quantized motional harmonic oscillator) states of a single trapped atom. Although this minimal system consists of only two qubits, it illustrates the basic operations necessary for, and the problems associated with, constructing a large scale quantum computer.

The distinctive feature of a quantum computer is its ability to store and process superpositions of numbers [2]. This potential for parallel computing has led to the discovery that certain problems are more efficiently solved on a quantum computer than on a classical computer [3]. The most dramatic example is an algorithm presented by Shor [4] showing that a quantum computer should be able to factor large numbers very efficiently. This appears to be of considerable interest, since the security of many data encryption schemes [5] relies on the inability of classical computers to factor large numbers.

A quantum computer hosts a register of qubits, each of which behaves as quantum mechanical two-level systems and can store arbitrary superposition states of 0 and 1 . It has been shown that any computation on a register of qubits can be broken up into a series of two-bit operations [6], for example, a series of two-bit "controlled-NOT" (CN) quantum logic gates, accompanied by simple rotations on single qubits $[7,8]$. The $\mathrm{CN}$ gate transforms the state of two qubits $\epsilon_{1}$ and $\epsilon_{2}$ from $\left|\epsilon_{1}\right\rangle\left|\epsilon_{2}\right\rangle$ to $\left|\epsilon_{1}\right\rangle\left|\epsilon_{1} \oplus \epsilon_{2}\right\rangle$, where the $\oplus$ operation is addition modulo 2. Reminiscent of the classical exclusive-OR (XOR) gate, the CN gate represents a computation at the most fundamental level: the "target" qubit $\left|\epsilon_{2}\right\rangle$ is flipped depending on the state of the "control" qubit $\left|\epsilon_{1}\right\rangle$.

Experimental realization of a quantum computer requires isolated quantum systems that act as the qubits, and the presence of controlled unitary interactions between the qubits that allow construction of the $\mathrm{CN}$ gate. As pointed out by many authors $[6,9,10]$, if the qubits are not sufficiently isolated from outside influences, decoherences can destroy the quantum interferences that form the computation. Several proposed experimental schemes for quantum computers and $\mathrm{CN}$ gates involving a dipole-dipole interaction between quantum dots or atomic nuclei $[6,7,11,12]$ may suffer from decoherence efforts. The light shifts on atoms located inside electromagnetic cavities have been shown to be large enough $[13,14]$ that one could construct a quantum gate where a single photon prepared in the cavity acts as the control qubit $[7,15]$ for the atomic state. However, extension to large quantum registers may be difficult. Cirac and Zoller [1] have proposed a very attractive quantum computer architecture based on laser-cooled trapped ions in which the qubits are associated with internal states of the ions, and information is transferred between qubits through a shared motional degree of freedom. The highlights of their proposal are that (i) decoherence can be small, (ii) extension to large registers is relatively straightforward, and (iii) the qubit readout can have nearly unit efficiency.

In our implementation of a quantum $\mathrm{CN}$ logic gate, the target qubit $|S\rangle$ is spanned by two ${ }^{2} S_{1 / 2}$ hyperfine ground states of a single ${ }^{9} \mathrm{Be}^{+}$ion (the $\left|f=2, m_{F}=2\right\rangle$ and $\left|F=1, m_{F}=1\right\rangle$ states, abbreviated by the equivalent spin-1/2 states $|\downarrow\rangle$ and $|\uparrow\rangle$ ) separated in frequency by $\omega_{0} / 2 \pi \simeq 1.250 \mathrm{GHz}$. The control qubit $|n\rangle$ is spanned by the first two quantized harmonic oscillator states of the trapped in $(|0\rangle$ and $|1\rangle)$, separated in frequency by the vibrational frequency $\omega_{x} / 2 \pi \simeq 11 \mathrm{MHz}$ of the harmonically trapped ion. Figure 1 displays the relevant ${ }^{9} \mathrm{Be}^{+}$energy levels. Manipulation between the four basis eigenstates spanning the two-qubit register $(|n\rangle|S\rangle=$ $|0\rangle|\downarrow\rangle,|0\rangle|\uparrow\rangle,|1\rangle|\downarrow\rangle,|1\rangle|\uparrow\rangle)$ is achieved by applying a pair of off-resonant laser beams to the ion, which drives stimulated Raman transitions between basis states. When the difference frequency $\delta$ of the beams is set near $\delta \simeq$ $\omega_{0}$ (the carrier), transitions are coherently driven between internal states $|S\rangle$ while preserving $|n\rangle$. Likewise, for $\delta \simeq$ $\omega_{0}-\omega_{x}$ (the red sideband), transitions are coherently driven between $|1\rangle|\downarrow\rangle$ and $|0\rangle|\uparrow\rangle$, and for $\delta \simeq \omega_{0}+\omega_{x}$ (the blue sideband), transitions are coherently driven between $|0\rangle|\downarrow\rangle$ and $|1\rangle|\uparrow\rangle$. Note that when $\delta$ is tuned to either sideband, the stimulated Raman transitions entangle $|S\rangle$ with $|n\rangle$, a crucial part of the trapped-ion quantum $\mathrm{CN}$ gate.

We realize the controlled-NOT gate by sequentially applying three pulses of the Raman beams to the ion 


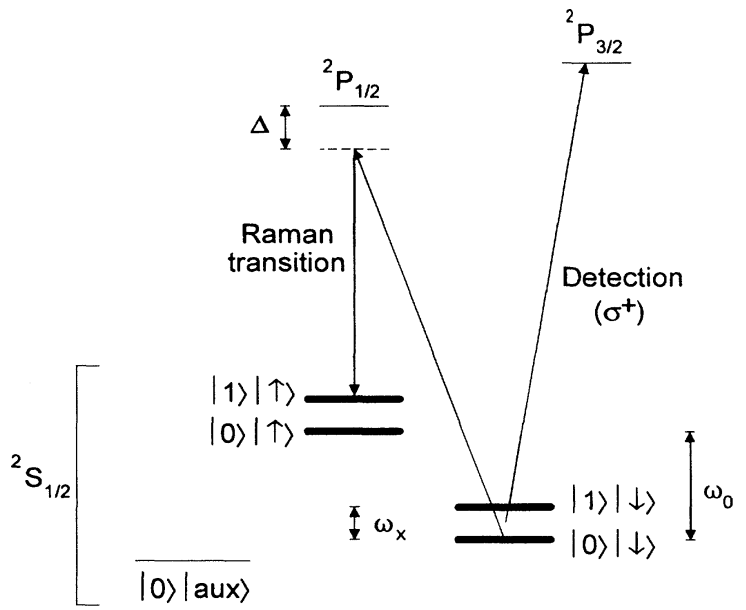

FIG. 1. ${ }^{9} \mathrm{Be}^{+}$energy levels. The levels indicated with thick lines form the basis of the quantum register: internal levels are $|S\rangle=|\downarrow\rangle$ and $|\uparrow\rangle\left({ }^{2} S_{1 / 2}\left|F=2, m_{F}=2\right\rangle\right.$ and ${ }^{2} S_{1 / 2}\left|F=1, m_{F}=1\right\rangle$ levels, respectively, separated by $\left.\omega_{0} / 2 \pi \simeq 1.250 \mathrm{GHz}\right)$, and $|\mathrm{aux}\rangle={ }^{2} S_{1 / 2}\left|F=2, m_{F}=0\right\rangle$ (separated from $|\downarrow\rangle$ by $\simeq 2.5 \mathrm{MHz}$ ); external vibrational levels are $|n\rangle=|0\rangle$ and $|1\rangle$ (separated by $\omega_{x} / 2 \pi \simeq 11.2 \mathrm{MHz}$ ). Stimulated Raman transitions between ${ }^{2} S_{1 / 2}$ hyperfine states are driven through the virtual ${ }^{2} P_{1 / 2}$ level $(\Delta \simeq 50 \mathrm{GHz})$ with a pair of $\simeq 313 \mathrm{~nm}$ laser beams. Measurement of $S$ is accomplished by driving the cycling $|\downarrow\rangle \rightarrow{ }^{2} P_{3 / 2}\left|F=3, m_{F}=3\right\rangle$ transition with $\sigma^{+}$-polarized light and detecting the resulting ion fluorescence.

according to the following format:

(a) A $\pi / 2$ pulse is applied on the carrier transition. The effect is described by the operator $V^{1 / 2}(\pi / 2)$ in the notation of Ref. [1].

(b) A $2 \pi$ pulse is applied on the blue sideband transition between $|\uparrow\rangle$ and an auxiliary atomic level $\mid$ aux $\rangle$ (see Fig. 1).

(c) A $\pi / 2$ pulse is applied on the carrier transition, with a $\pi$ phase shift relative to (a), leading to the operator $V^{1 / 2}(-\pi / 2)$ of Ref. [1].

The $\pi / 2$ pulses in steps (a) and (c) cause the spin $|S\rangle$ to undergo $+1 / 4$ and $-1 / 4$ of a complete Rabi cycle, respectively, while leaving $|n\rangle$ unchanged. The auxiliary transition in step (b) simply reverses the sign of any component of the register in the $|1\rangle|\uparrow\rangle$ state by inducing a complete Rabi cycle from $|1\rangle|\uparrow\rangle \rightarrow|0\rangle \mid$ aux $\rangle \rightarrow-|1\rangle|\uparrow\rangle$. The auxiliary level $\mid$ aux $\rangle$ is the ${ }^{2} S_{1 / 2}\left|F=2, m_{F}=0\right\rangle$ ground state, split from the $|\downarrow\rangle$ state by virtue of a Zeeman shift of $\simeq 2.5 \mathrm{MHz}$ resulting from a $0.18 \mathrm{mT}$ applied magnetic field (see Fig. 1). Any component of the quantum register in the $|n\rangle=|0\rangle$ state is unaffected by the blue sideband transition of step (b), and the effects of the two Ramsey $\pi / 2$ pulses cancel. On the other hand, any component of the quantum register in the $|1\rangle|\uparrow\rangle$ state acquires a sign change in step (b), and the two Ramsey pulses add constructively, effectively "flipping" the target qubit by $\pi$ radians. The truth table of the $\mathrm{CN}$ operation is as follows:

$$
\begin{gathered}
\text { Input state } \rightarrow \text { Output state } \\
|0\rangle|\downarrow\rangle \rightarrow|0\rangle|\downarrow\rangle \\
|0\rangle|\uparrow\rangle \rightarrow|0\rangle|\uparrow\rangle \\
|1\rangle|\downarrow\rangle \rightarrow|1\rangle|\uparrow\rangle \\
|1\rangle|\uparrow\rangle \rightarrow|1\rangle|\downarrow\rangle .
\end{gathered}
$$

The experiment apparatus is described elsewhere [16,17]. A single ${ }^{9} \mathrm{Be}^{+}$ion is stored in a coaxialresonator rf-ion trap [17], which provides pseudopotential oscillation frequencies of $\left(\omega_{x}, \omega_{y}, \omega_{z}\right) / 2 \pi \simeq(11.2,18.2$, 29.8) $\mathrm{MHz}$ along the principal axes of the trap. We cool the ion so that the $n_{x}=0$ vibrational ground state is occupied $\simeq 95 \%$ of the time by employing resolved-sideband stimulated Raman cooling in the $x$ dimension, exactly as in Ref. [16]. The two Raman beams each contain $\simeq 1 \mathrm{~mW}$ of power at $\simeq 313 \mathrm{~nm}$ and are detuned $\simeq 50 \mathrm{GHz}$ red of the ${ }^{2} P_{1 / 2}$ excited state. The Raman beams are applied to the ion in directions such that their wave-vector difference $\boldsymbol{\delta} \mathbf{k}$ points nearly along the $x$ axis of the trap; thus the Raman transitions are highly insensitive to motion in the other two dimensions. The Lamb-Dicke parameter is $\eta_{x}=\delta k x_{0} \simeq 0.2$, where $x_{0} \simeq 7 \mathrm{~nm}$ is the spread of the $n_{x}=0$ wave function. The carrier $(|n\rangle|\downarrow\rangle \rightarrow|n\rangle|\uparrow\rangle)$ Rabi frequency is $\Omega_{0} 2 \pi \simeq 140 \mathrm{kHz}$, the red $(|1\rangle|\downarrow\rangle \rightarrow|0\rangle|\uparrow\rangle)$ and blue $(|0\rangle|\downarrow\rangle \rightarrow|1\rangle|\uparrow\rangle)$ sideband Rabi frequencies are $\eta_{x} \Omega_{0} / 2 \pi \simeq 30 \mathrm{kHz}$, and the auxiliary transition $(|1\rangle|\uparrow\rangle \rightarrow|0\rangle|\downarrow\rangle)$ Rabi frequency is $\eta_{x} \Omega_{\mathrm{aux}} / 2 \pi \simeq 12 \mathrm{kHz}$. The difference frequency of the Raman beams is tunable from 1200 to $1300 \mathrm{MHz}$ with the use of a double pass acousto-optic modulator (AOM), and the Raman pulse durations are controlled with additional switching AOMs. Since the Raman beams are generated from a single laser and an AOM, broadening of the Raman transitions due to a finite laser linewidth is negligible [18].

Following Raman cooling to the $|0\rangle|\downarrow\rangle$ state, but before application of the $\mathrm{CN}$ operation, we apply appropriately tuned and timed Raman pulses to the ion, which can prepare an arbitrary state of the two-qubit register. For instance, to prepare a $|1\rangle|\downarrow\rangle$ eigenstate, we apply a $\pi$ pulse on the blue sideband followed by a $\pi$ pulse on the carrier $(|0\rangle|\downarrow\rangle \rightarrow|1\rangle|\uparrow\rangle \rightarrow|1\rangle|\downarrow\rangle)$. We perform two measurements to detect the population of the register after an arbitrary sequence of operations. First, we measure the probability $P\{S=\downarrow\}$ that the target qubit $|S\rangle$ is in the $|\downarrow\rangle$ state by collecting the ion fluorescence when $\sigma^{+}$-polarized laser radiation is applied resonant with the cycling $|\downarrow\rangle \rightarrow{ }^{2} P_{3 / 2}\left|F=3, m_{F}=3\right\rangle$ transition (radiative linewidth $\gamma / 2 \pi \simeq 19.4 \mathrm{MHz}$ at $\lambda \simeq 313 \mathrm{~nm}$; see Fig. 1). Since this radiation does not appreciably couple to the $|\uparrow\rangle$ state (relative excitation probability: $\simeq 5 \times 10^{-5}$ ), the fluorescence reading is proportional to $P\{S=\downarrow l$. For $S=\downarrow$, we collect on average $\simeq 1$ photon per measurement cycle [16]. Once $S$ is measured, we 
perform a second independent measurement that provides the probability $P\{n=1\}$ that the control bit $|n\rangle$ is in the 11) state: After the same operation sequence is repeated, an appropriate Raman pulse is added just prior to the detection of $S$. This "detection preparation" pulse maps $|n\rangle$ onto $|S\rangle$. For instance, if we first measure $S$ to be $\downarrow$, we repeat the experiment with the addition of a " $\pi$ pulse" on the red sideband. Subsequent detection of $S$ resulting in the presence (absence) of fluorescence indicates that $n=0$ (1). Likewise, if we first measure $S$ to be $\uparrow$, we repeat the experiment with the addition of a " $\pi$ pulse" on the blue sideband. Subsequent detection of $S$ resulting in the presence (absence) of fluorescence indicates that $n=1(0)$.

In the above measurement scheme, we do not obtain phase information about the quantum state of the register and therefore do not measure the complete transformation matrix associated with the $\mathrm{CN}$ operation. The phase information could be obtained by performing additional operations (similar to those described above) prior to the measurement of $S$. Here, we demonstrate the key features of the $\mathrm{CN}$ gate by (i) verifying that the populations of the register follow the truth table given in (2), and (ii) demonstrating the conditional quantum dynamics associated with the $\mathrm{CN}$ operation.

To verify the $\mathrm{CN}$ truth table, we separately prepare each of the four eigenstates spanning the register $(|n\rangle|S\rangle=$ $|0\rangle|\downarrow\rangle,|0\rangle|\uparrow\rangle,|1\rangle|\downarrow\rangle,|1\rangle|\uparrow\rangle)$, then apply the CN operation given in (1). We measure the resulting register population as described above after operation of the $\mathrm{CN}$ gate, as shown in Fig. 2. When the control qubit is prepared in the $|n\rangle=|0\rangle$ state, the measurements show that the gate preserves $S$ with high probability, whereas when the initial control qubit is prepared in the $|n\rangle=|1\rangle$ state, the $\mathrm{CN}$ gate flips the value of $S$ with high probability. In contrast, the gate preserves the population $n$ of the control qubit $|n\rangle$ with high probability, verifying that the register populations follow the $\mathrm{CN}$ truth table expressed in (2). The fact that the measured probabilities are not exactly zero or one is primarily due to imperfect laser-cooling, imperfect state preparation and detection preparation, and decoherence effects.

To illustrate the conditional dynamics of a quantum logic gate, we desire to perform a unitary transformation on one physical system conditioned upon the quantum state of another subsystem [19]. To see this in the present experiment, it is useful to view steps (a) and (c) of the CN operations given in (1) as Ramsey radiation pulses [20], which drive the $|n\rangle|\downarrow\rangle \rightarrow|n\rangle|\uparrow\rangle$ transitionwith the addition of the perturbation (b) inserted between the pulses. If we now vary the frequency of the Ramsey pulses, we obtain the typical sinusoidal Ramsey interference pattern indicative of the coherent evolution between states $|S\rangle=|\downarrow\rangle$ and $|\uparrow\rangle$. However, the final population $S$ depends on the status of the control qubit $|n\rangle$. This is illustrated in Fig. 3 where we plot the measured probability $P\{S=\downarrow\}$ as a function of detuning of the Ram-

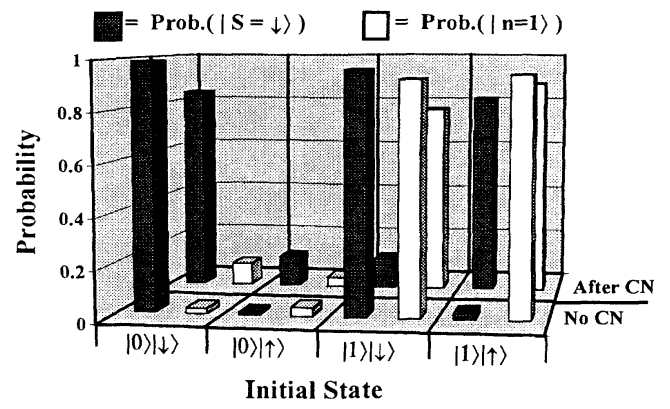

FIG. 2. Controlled-NOT (CN) truth table measurements for eigenstates. The two horizontal rows give measured final values of $n$ and $S$ with and without operation of the $C N$ gate, expressed in terms of the probabilities $P\{n=1\}$ and $P\{S=\downarrow\}$. The measurements are grouped according to the initial prepared eigenstate of the quantum register $(|0\rangle|\downarrow\rangle,|0\rangle|\uparrow\rangle,|1\rangle|\downarrow\rangle$, or $|1\rangle|\uparrow\rangle)$. Even without $\mathrm{CN}$ operations, the probabilities are not exactly 0 or 1 due to imperfect laser-cooling, state preparation and detection preparation, and decoherence effects. However, with high probability, the $\mathrm{CN}$ operation preserves the value of the control qubit $n$, and flips the value of the target qubit $S$ only if $n=1$.

sey pulses. For initial state $|0\rangle|\downarrow\rangle$, we obtain the normal Ramsey spectrum since step (b) is inactive. For initial state $|1\rangle|\downarrow\rangle$, the Ramsey spectrum is inverted indicating the conditional control (by quantum bit $|n\rangle$ ) of the dynamics of the Ramsey pulses. Appropriate Ramsey curves are also obtained for initial states $|0\rangle|\uparrow\rangle$ and $|1\rangle|\uparrow\rangle$.

The switching speed of the CN gate is approximately $20 \mathrm{kHz}$, limited mainly by the auxiliary $2 \pi$ pulse in step (b) given in (1). This rate could be increased with more Raman beam laser power, although a fundamental limit in switching speed appears to be the frequency separation of the control qubit vibrational energy levels, which can be as high as $50 \mathrm{MHz}$ in our experiment [17].

We measure a decoherence rate of a few $\mathrm{kHz}$ in the experiment, adequate for a single $\mathrm{CN}$ gate operating at a speed of $\simeq 20 \mathrm{kHz}$, but certainly not acceptable for a more extended computation. We identify several sources responsible for decoherence, including instabilities in the laser beam power and the relative position of the ion with respect to the beams, fluctuating external magnetic fields (which can modulate the qubit phases), and instabilities in the rf-ion trap drive frequency and voltage amplitude. Substantial reduction of these sources of decoherence can be expected. Other sources of decoherence that may become important in the future include external heating and dissipation of the ion motion [16,21], and spontaneous emission caused by off-resonant transitions. We note that decoherence rates of under $0.001 \mathrm{~Hz}$ have been achieved for internal-state ion qubits [22].

The single-ion quantum register in the experiment comprises only two qubits and is therefore not useful for computation. However, if the techniques described here are applied to a collection of many ions cooled to the $n=0$ state of collective motion, it should be possible to imple- 


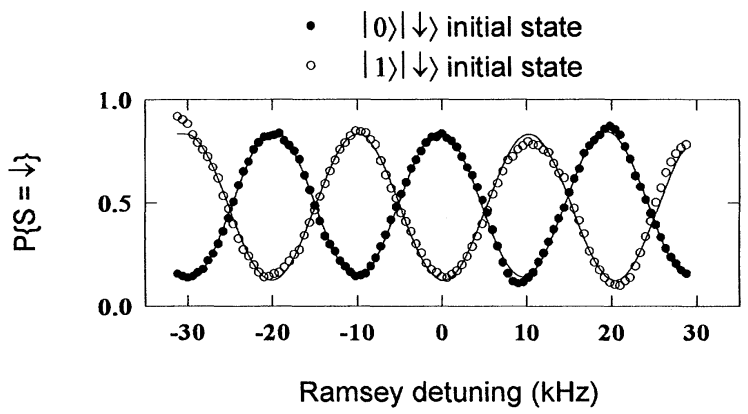

FIG. 3. Ramsey spectra of the controlled-NOT (CN) gate. The detuning of the Ramsey $\pi / 2$ pulses of the $\mathrm{CN}$ gate [steps (a) and (c)] is swept, and $S$ is measured, expressed in terms of the probability $P\{S=\downarrow\}$. The solid points correspond to initial preparation in the $|n\rangle|S\rangle=|0\rangle|\downarrow\rangle$ state, and the hollow points correspond to preparation in the $|n\rangle|S\rangle=|1\rangle|\downarrow\rangle$ state. The resulting patterns are shifted in phase by $\pi \mathrm{rad}$. This flipping of $|S\rangle$ depending on the state of the control qubit indicates the conditional dynamics of the gate. Similar curves are obtained when the $|n\rangle|S\rangle=|0\rangle|\uparrow\rangle$ and $|1\rangle|\uparrow\rangle$ states are prepared. The lines are fits by a sinusoid, and the width of the Ramsey fringes is consistent with the $\simeq 50 \mu \mathrm{sec}$ duration of the $\mathrm{CN}$ operation.

ment computations on larger quantum registers. For example, the CN gate between two ions ( $m$ and $n$ ) might be realized by mapping the internal state of the $m$ th ion onto the collective vibrational state of all ions, applying the single-ion $\mathrm{CN}$ operation demonstrated in this work to the $n$th ion, then returning the vibrational state back to the internal state of the $m$ th ion. (This mapping may be achieved by simply driving a $\pi$ pulse on the red of blue sideband of the $m$ th ion.) This approach is equivalent to the scheme proposed by Cirac and Zoller [1,23]. An arbitrary computation may then be broken into a number of such operations on different pairs of ions, accompanied by single qubit rotations on each ion (carrier transitions) [6-8].

We are currently devoting effort into the multiplexing of the register to many ions. Several technical issues remain to be explored in this scaling, including lasercooling efficiency, the coupling of internal vibrational modes due to trap imperfections, and the unique addressing of each ion with laser beams. Although we can trap and cool a few ions in the current apparatus, other geometries such as the linear rf-ion trap [24] or an array of ion traps each confining a single ion [25] might be considered.

This work is supported by the U.S. Office of Naval Research and the U.S. Army Research Office. We acknowledge useful contributions from J.C. Bergquist and J. J. Bollinger. We thank Robert Peterson, Dana Berkeland, and Chris Myatt for helpful suggestions on the manuscript.
[1] J. I. Cirac and P. Zoller, Phys. Rev. Lett. 74, 4091 (1995).

[2] R.P. Feynman, Int. J. Theor. Phys. 21, 467 (1982); Opt. News 11, 11 (1985).

[3] D. Deutsch, Proc. R. Soc. London A 425, 73 (1989); D. Deutsch and R. Jozsa, Proc. Soc. London A 439, 554 (1992).

[4] P. Shor, in Proceedings of the 35th Annual Symposium on the Foundations of Computer Science (IEEE Computer Society Press, New York, 1994), p. 124.

[5] R. L. Rivest, A. Shamir, and L. Adelman, Comm. ACM, 28, 120 (1978).

[6] D. P. DiVincenzo, Phys. Rev. A 51, 1015 (1995).

[7] A. Barenco, D. Deutsch, A. Ekert, and R. Jozsa, Phys. Rev. Lett. 74, 4083 (1995).

[8] A. Barenco, C.H. Bennett, R. Cleve, D. P. DiVincenzo, N. Margolus, P. Shor, T. Sleator, J. Smolin, and H. Weinfurter, Phys. Rev. A 52, 3457 (1995).

[9] R. Landauer, in Proceedings of the Drexel-4 Symposium on Quantum Nonintegrability-Quantum Classical Correspondence, edited by D.H. Feng and B-L. Hu (International Press, Boston, to be published).

[10] W. G. Unruh, Phys. Rev. A 51, 992 (1995).

[11] K. Obermeyer, W. G. Teich, and G. Mahler, Phys. Rev. B 37, 8111 (1988).

[12] S. Lloyd, Science 261, 1569 (1993).

[13] M. Brune, P. Nussenzveig, F. Schmidt-Kaler, F. Bernardot, A. Maali, J. M. Raimond, and S. Haroche, Phys. Rev. Lett. 72, 3339 (1994); M. Brune, F. Schmidt-Kaler, J. Deyer, A. Maali, and J. M. Raimond, "Laser Spectroscopy $X I I$," edited by M. Inguscio (World Scientific, Singapore, to be published).

[14] H. J. Kimble, in “Laser Spectroscopy XIII" Ref. [13].

[15] T. Sleator and H. Weinfurter, Phys. Rev. Lett. 74, 4087 (1995).

[16] C. Monroe, D. M. Meekhof, B. E. King, S. R. Jefferts, W. M. Itano, D. J. Wineland, and P. Gould, Phys. Rev. Lett. 75, 4011 (1995).

[17] S. R. Jefferts, C. Monroe, E. W. Bell, and D. J. Wineland, Phys. Rev. A 51, 3112 (1995).

[18] J. E. Thomas, P. R. Hemmer, S. Ezekiel, C. C. Leiby, R. H. Picard, and C. R. Willis, Phys. Rev. Lett. 48, 867 (1982).

[19] A. Ekert and R. Jozsa, Rev. Mod. Phys. (to be published).

[20] N. F. Ramsey, Molecular Beams (Oxford University Press, London, 1956).

[21] F. Diedrich, J.C. Bergquist, W. M. Itano, and D. J. Wineland, Phys. Rev. Lett. 62, 403 (1989).

[22] J. J. Bollinger, D. J. Heinzen, W. M. Itano, S. L. Gilbert, and D. J. Wineland, IEEE Trans. Instrum. Meas. 40, 126 (1991).

[23] The controlled-NOT operator proposed in Ref. [1] is $V_{n}^{1 / 2}(\pi / 2) U_{m}^{1,0} U_{n}^{2,1} U_{m}^{1,0} V_{n}^{1 / 2}(-\pi / 2)$, adopting their notation. This is equivalent to the controlled-NOT operator proposed here between ions $m$ and $n$, $U_{m}^{1,0} V_{n}^{1 / 2}(\pi / 2) U_{n}^{2,1} V_{n}^{1 / 2}(-\pi / 2) U_{m}^{1,0}, \quad$ since $V_{n} \quad$ and $U_{m}$ commute.

[24] M. G. Raizen, J. M. Gilligan, J. C. Bergquist, W. M. Itano, and D. J. Wineland, Phys. Rev. A 45, 6493 (1992).

[25] F. Major, J. Phys. (Paris) Lett. 38, L221 (1977); R. Brewer, R. G. DeVoe, and R. Kallenbach, Phys. Rev. A 46, R6781 (1992). 


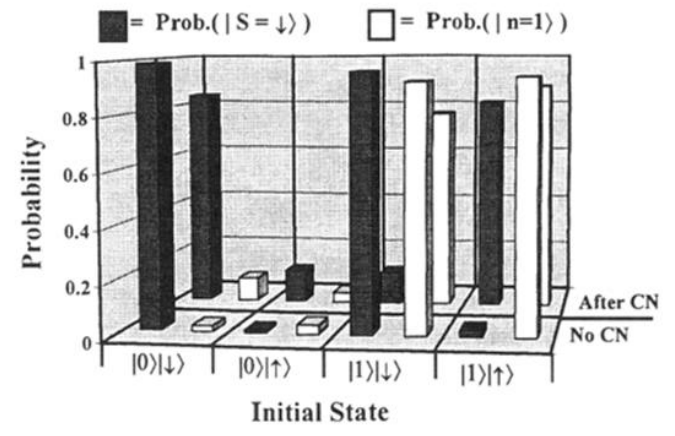

FIG. 2. Controlled-NOT $(\mathrm{CN})$ truth table measurements for eigenstates. The two horizontal rows give measured final values of $n$ and $S$ with and without operation of the $C N$ gate, expressed in terms of the probabilities $P\{n=1\}$ and $P\{S=1\}$. The measurements are grouped according to the initial prepared eigenstate of the quantum register $(|0\rangle|\downarrow\rangle,|0\rangle|\uparrow\rangle,|1\rangle|\downarrow\rangle$, or $|1\rangle|\uparrow\rangle)$. Even without $\mathrm{CN}$ operations, the probabilities are not exactly 0 or 1 due to imperfect laser-cooling, state preparation and detection preparation, and decoherence effects. However, with high probability, the $\mathrm{CN}$ operation preserves the value of the control qubit $n$, and flips the value of the target qubit $S$ only if $n=1$. 